\title{
Computing supersonic non-premixed turbulent combustion by an SMLD flamelet progress variable model
}

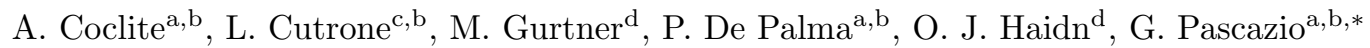 \\ ${ }^{a}$ Dipartimento di Meccanica, Matematica e Management (DMMM), Politecnico di Bari, Via Re David 200 - 70125 Bari, \\ Italy \\ ${ }^{b}$ Centro di Eccellenza in Meccanica Computazionale (CEMeC), Politecnico di Bari, Via Re David 200 - 70125 Bari, Italy \\ ${ }^{c}$ Centro Italiano Ricerche Aerospaziali (CIRA), via Maiorise - 81043 Capua, Italy \\ ${ }^{d}$ Institute for Flight Propulsion (LFA), Technische Universität München, Boltzmannstrasse 15 - 85748, Garching, Germany
}

\begin{abstract}
This paper describes the numerical simulation of the NASA Langley Research Center supersonic $\mathrm{H}_{2}$-Air combustion chamber performed using two approaches to model the presumed probability density function (PDF) in the flamelet progress variable (FPV) framework. The first one is a standard FPV model, built presuming the functional shape of the PDFs of the mixture fraction, $Z$, and of the progress parameter, $\Lambda$. In order to enhance the prediction capabilities of such a model in high-speed reacting flows, a second approach is proposed employing the statistically most likely distribution (SMLD) techcnique to presume the joint PDF of $Z$ and $\Lambda$, without any assumption about their behaviour. The standard and FPV-SMLD models have been developed using the low Mach number assumption. In both cases, the temperature is evaluated by solving the total-energy conservation equation, providing a more suitable approach for the simulation of supersonic combustion. By comparison with experimental data, the proposed SMLD model is shown to provide a clear improvement with respect to the standard FPV model, especially in the auto-ignition and stabilization regions of the flame.
\end{abstract}

Keywords: Joint Presumed PDF modelling, Hydrogen-Air combustion, Reynolds-Averaged Navier-Stokes equations

\section{Nomenclature}

$C$ Progress variable

$D_{\phi}$ Diffusivity of $\phi$

$H$ Total enthalpy

$k$ Turbulent kinetic energy

Ma Mach number of the flow

$p$ Pressure

$\widetilde{P}(x)$ Density-weighted probability density function
$\widetilde{P}_{S M L, 2}(x)$ Density-weighted probability density function evaluated as the statistical most likely distribution with the second order moments

$q$ Heat flux

$\dot{q}_{\text {react }}$ Heat release rate

$R$ Gas constant

Re Reynolds number of the flow

$T$ Temperature

Tu Turbulence intensity

\footnotetext{
* Corresponding author

Email addresses: alessandro.coclite@poliba.it (A. Coclite), 1.cutrone@cira.it (L. Cutrone), gurtner@lfa.mw.tum.de (M. Gurtner), pietro.depalma@poliba.it (P. De Palma), haidn@lfa.mw.tum.de (O. J. Haidn), giuseppe.pascazio@poliba.it (G. Pascazio)
} 
$u$ Velocity

$Y_{\phi}$ Mass fraction of $\phi$

$Z$ Mixture fraction

$\beta(x) \beta$-distribution

$\Gamma$ Euler function

$\delta(x)$ Dirac distribution

$\Lambda$ Progress parameter

$\mu$ Dynamic viscosity

$\mu_{x}$ Lagrangian multiplier

$\nu$ Kinematic viscosity

$\rho$ Density

$\phi$ Generic thermo-chemical quantity $\widetilde{\phi}$ Favre-averaged value of $\phi$

$\phi^{\prime \prime}$ Fluctuation of $\phi$ in the Favre-averaging process

$\widetilde{\phi^{\prime \prime 2}}$ Variance of $\phi$

$\bar{\phi}$ Reynolds-averaged value of $\phi$

$\phi^{\prime}$ Fluctuation of $\phi$ in the Reynolds-averaging process

$\Phi$ Error function

$\chi$ Scalar dissipation rate

$\chi_{s t}$ Value of $\chi$ at the stoichiometric point

$\omega$ Turbulent kinetic energy specific dissipation rate

$\dot{\omega}_{\phi}$ Production therm of $\phi$

\section{Introduction}

In the last years, the development of propulsion systems based on air-breathing engines for super-/hypersonic flying vehicles has fostered the study of supersonic combustion, see, e.g., the review of Cecere et al. [1]. In these systems, hydrogen is one of the preferred fuel because of its properties in terms of very short ignition delay time and high energy per unit weight. The investigation of hydrogen supersonic combustion presents significant difficulties and high costs either following the experimental approach or the numerical one. In fact, in supersonic combustion, the mixing time scales are comparable to $H_{2}$-Air reaction time scales 2 . Moreover, high-Reynolds-number turbulent combustion is a formidable multi-scale problem, where the interaction between chemical kinetics, molecular, and turbulent transport occurs over a wide range of length and time scales. These features pose severe difficulties in the analysis and comprehension of the basic phenomena involved in supersonic combustion.

Concerning the numerical approach, in recent years, the need for efficient tools having affordable computational costs has driven the research towards: i) studying turbulent combustion in order to understand the interaction between turbulence and chemistry [3, 4, 5, 6]; ii) improving kinetic schemes to describe the combustion process [7, 8, 9. Higher accuracy can be achieved by employing models based on detailed kinetic mechanisms, but this usually leads to prohibitively expensive calculations. Therefore, reduced models are often employed to condensate the reaction mechanisms and cut down the computational costs [7]. Simplified approaches to combustion modelling have been proposed to further reduce the number of equations to be solved; for instance, the reduction of the chemical scheme in intrinsic low dimensional manifolds (ILDM) [10] the flamelet-based approaches such as the flamelet-progress variable (FPV) [1] or flame prolongation of ILDM (FPI) [12; and flamelet generated manifolds approach (FGM) [13].

The present work is based on the FPV model for non-premixed flames. Standard steady FPV models are built under the low Mach number hypothesis [14, 15, 16, 17, computing the combustion process at a uniform given pressure. Obviously, in supersonic combustion, density variations due to the dynamics of the flow cannot be neglected. For this reason, here we employ the modified FPV approach proposed by Oevermann [18, and recently employed by other authors [19, where the fluid temperature is not obtained from the flamelet libraries as in the standard model, but is calculated by solving the full set of conservation equations for compressible flows, including the energy equation. More recent works have proposed an extension of FPV combustion models to compressible flows by using a source term linearly rescaled with the pressure value and a perturbation of the low-Mach number flamelet accounting for compressibility [20, 21]. 
For the case of non-premixed combustion of interest here, mixing must bring reactants into the reaction zone so as to activate and maintain the combustion process. Such flames are characterized by a local balance between diffusion and reaction [22. The basic assumption of the flamelet model is that each element of the flame front can be described as a small laminar flame, also called flamelet. Therefore, for a steady flow, the flame structure can be described as a function of the mixture fraction, $Z$, and of the progress parameter, $\Lambda$ [23]. For turbulent combustion, a probability density function (PDF) is needed to compute the mean value and the variance of the thermo-chemical variables. The definition of such a PDF is critical since it has a strong impact on the solution. The aim of this work is to study the applicability of the statistically most likely distribution (SMLD) 24] approach to model joint-PDF of $Z$ and $\Lambda$ in the case of supersonic combustion. The proposed joint-SMLD approach is very interesting since it represents a good compromise between computational costs and accuracy level. The results obtained using the proposed model are validated versus experimental data and compared with numerical results available in the literature as well as with those obtained using the standard FPV model.

This work is organised as follow: sections 2 and 3 provide the theoretical description and some details of the numerical discretization for the two models. The comparison between their numerical results for the simulation of the NASA Langley Research Center supersonic hydrogen flame are presented in section 4 along with reference numerical and experimental data. Finally, some conclusions are provided.

\section{The flamelet progress variable models}

For the case of non-premixed combustion of interest here, the basic assumptions of the flamelet model are fulfilled for sufficiently large Damköhler number, $D a$. In fact, when the reaction zone thickness is very thin with respect to the Kolmogorov length scale, turbulent structures are unable to penetrate into the reaction zone and cannot destroy the laminar flame structure. Effects of turbulence only result in a deformation and straining of the flame sheet and locally the flame structure can be described as function of the mixture fraction, $Z$, the scalar dissipation rate, $\chi$, and the time. The scalar dissipation rate, $\chi=2 D_{Z}(\nabla Z)^{2}$, is a measure of the gradient of the mixture fraction representing the molecular diffusion of the species in the flame, $D_{Z}$ being the molecular diffusion coefficient of the chemical species. Therefore, the entire flame behaviour can be obtained as a combination of solutions of the laminar flamelet equation. In the present work we consider a further simplification assuming a steady flamelet behaviour, so that chemical effects are entirely determined by the value of $Z$, whereas $\chi$ describes the effects of the flow on the flame structure according to the following steady laminar flamelet equation (SLFE) for the generic variable $\phi$ :

$$
-\rho \frac{\chi}{2} \frac{\partial^{2} \phi}{\partial Z^{2}}=\dot{\omega}_{\phi}
$$

In equation (1), $\rho$ is the density and $\dot{\omega}_{\phi}$ is the source term related to $\phi[23$, different from zero in the case of finite rate chemistry. In particular, in this work, the FPV model proposed by Pierce and Moin [11, 23] is employed to evaluate all of the thermo-chemical quantities involved in the combustion process. This approach is based on the parametrization of the generic thermo-chemical quantity, $\phi$, in terms of the mixture fraction, $Z$, and of the progress parameter, $\Lambda$, instead of $\chi$ :

$$
\phi=F_{\phi}(Z, \Lambda) \text {. }
$$

Using such a parameter, independent of the mixture fraction, one can uniquely identify each flame state along the stable and unstable branches of the S-shaped curve. A suitable definition of $\Lambda$ leads to a dramatic simplification of the presumed PDF closure model. On the other hand, the solution of the transport equation for $\Lambda$ is quite complex since it requires non-trivial modelling of several unclosed terms [25]. In order to overcome such a difficulty, the progress parameter is derived from a reaction progress variable, $C$, such as the temperature or a linear combination of the main reaction products, whose behaviour is governed by a simpler transport equation. Therefore, a transport equation for $C$ is solved and the flamelet library is parametrized in terms of $Z$ and $C$. Requiring that the transformation between $\Lambda$ and $C$ be bijective, from equation (1) one has

$$
\Lambda=F_{C}^{-1}(Z, C)
$$


and any thermo-chemical variable can be expressed as:

$$
\phi=F_{\phi}\left(Z, F_{C}^{-1}(Z, C)\right) .
$$

The choice of the progress variable is not unique and some recent works discuss in details this issue proposing a procedure for its optimal selection [26, 27, 28]. A suitable definition for the progress variable is the sum of the mass fraction of the main products [26]; for hydrogen combustion [21]:

$$
\mathrm{C}=\mathrm{Y}_{\mathrm{H}_{2} \mathrm{O}} \text {. }
$$

A stretching with respect to the minimum and maximum conditioned value of $C$ over the mixture fraction is made, leading to the following form of the progress parameter:

$$
\Lambda=\frac{C-C_{M i n} \mid Z}{C_{M a x}\left|Z-C_{M i n}\right| Z} .
$$

Equation (2) is taken as the solution of the SLFE (1). Even if cases in which there is not a unique mapping of this solution in function of $Z$ and $\Lambda$ are reported in the literature [11, 23, such events are excluded from the solution family composing the flamelet library, since they are very close to the equilibrium limit. Since flamelet libraries are computed in advance and are assumed to be independent of the flow field, one has to model the dependence of $\chi$ on $Z$ [14, 29, 30. In this work, the functional form of $\chi(Z)$ has been taken from an idealized flow configuration, as proposed by Peters [31]; the distribution of the scalar dissipation rate in a counterflow diffusion flame, $\Phi(Z)$, is employed, scaled in the following way:

$$
\chi(Z)=\chi_{s t} \frac{\Phi(Z)}{\Phi\left(Z_{s t}\right)},
$$

where $\chi_{s t}$ and $Z_{s t}$ are evaluated at the stoichiometric point 31].

From equation (2) one can derive the generic thermo-chemical quantity $\phi$. When a turbulence model is used, the Favre-averaged value of $\phi$ and its variance are given as:

$$
\begin{gathered}
\widetilde{\phi}=\iint F_{\phi}(Z, \Lambda) \widetilde{P}(Z, \Lambda) d Z d \Lambda, \\
\widetilde{\phi^{\prime \prime 2}}=\iint\left(F_{\phi}(Z, \Lambda)-\widetilde{\phi}\right)^{2} \widetilde{P}(Z, \Lambda) d Z d \Lambda .
\end{gathered}
$$

In the above equations, $\widetilde{P}(Z, \Lambda)$ is the density-weighted $\mathrm{PDF}$,

$$
\widetilde{P}(Z, \Lambda)=\frac{\rho P(Z, \Lambda)}{\bar{\rho}},
$$

$P(Z, \Lambda)$ is the joint PDF and $\bar{\rho}$ is the Reynolds-averaged density. As usual, $\phi$ can be decomposed as:

$$
\phi=\widetilde{\phi}+\phi^{\prime \prime}, \quad \widetilde{\phi}=\frac{\overline{\rho \phi}}{\bar{\rho}}
$$

and

$$
\rho=\bar{\rho}+\rho^{\prime}
$$

where $\phi^{\prime \prime}$ and $\rho^{\prime}$ are the fluctuations. The joint-PDF, $\widetilde{P}(Z, \Lambda)$, plays a crucial role in the definition of the model, affecting both its accuracy and computational costs. Moreover, the choice of such a function is not straightforward because of the unknown statistical behaviour of the two variables $Z$ and $\Lambda$ [25] and its definition is still an open problem whose solution is being pursued by several researches [32, 33, 34, 35]. The aim of this work is to validate a more general model based on the statistically most likely distribution (SMLD) 24] for the joint PDF of $Z$ and $\Lambda$ [36]. The performance of such a combustion model are assessed by computing a hydrogen-air supersonic flame and comparing the results with those obtained using the standard FPV model. In the present work, the Reynolds-Averaged Navier-Stokes equations with $k-\omega$ turbulence model 37 are solved and both the standard-FPV and the FPV-SMLD models employ the total energy conservation equation to evaluate the temperature field in order to improve the simulation of compressible reacting flows. 


\subsection{Presumed probability density function model}

In this section, the standard FPV model 23] (called here model A) and the FPV-SMLD model (called here model B) 36 are briefly described.

The evaluation of the average quantities in equations $(8)$ and $(9)$ requires the PDF to be known or somehow presumed. Such a PDF establishes the statistical correlation between $Z$ and $\Lambda$. Employing the Bayes' theorem,

$$
\widetilde{P}(Z, \Lambda)=\widetilde{P}(Z) \widetilde{P}(\Lambda \mid Z)
$$

one usually presumes the functional shape of the marginal PDF of $Z$ and of the conditional PDF of $\Lambda \mid Z$. In model $\mathrm{A}$, the basic assumption is the statistical independence between $Z$ and $\Lambda$, so that $\widetilde{P}(Z, \Lambda)=$ $\widetilde{P}(Z) \widetilde{P}(\Lambda)$. Furthermore, the statistical behaviour of the mixture fraction is described by a $\beta$-distribution. In fact, even though the definition of $\widetilde{P}(Z)$ is still an open question [24, it has been shown by several authors that the mixture fraction behaves like a passive scalar whose statistical distribution can be approximated by a $\beta$ function $[38,39,40$. The two-parameter family of the $\beta$-distribution in the interval $x \in[0,1]$ is given by:

$$
\beta\left(x ; \widetilde{x}, \widetilde{x^{\prime \prime 2}}\right)=x^{a-1}(1-x)^{b-1} \frac{\Gamma(a+b)}{\Gamma(a) \Gamma(b)},
$$

where $\Gamma(x)$ is the Euler function and $a$ and $b$ are two parameters related to $\widetilde{x}$ and $\widetilde{x^{\prime \prime 2}}$

$$
a=\frac{\widetilde{x}\left(\widetilde{x}-\widetilde{x}^{2}-\widetilde{x^{\prime \prime 2}}\right)}{\widetilde{x^{\prime \prime 2}}}, \quad b=\frac{(1-\widetilde{x})\left(\widetilde{x}-\widetilde{x}^{2}-\widetilde{x^{\prime \prime 2}}\right)}{\widetilde{x^{\prime \prime 2}}} .
$$

Moreover, $\widetilde{P}(\Lambda)$ is chosen as a Dirac distribution, implying a great simplification in the theoretical framework. With these assumptions, the Favre-average of a generic thermo-chemical quantity is given by:

$$
\widetilde{\phi}=\iint F_{\phi}(Z, \Lambda) \widetilde{\beta}(Z) \delta(\Lambda-\widetilde{\Lambda}) d Z d C=\int F_{\phi}(Z, \widetilde{\Lambda}) \widetilde{\beta}(Z) d Z .
$$

Therefore, in addition to the conservation and turbulence model equations, one has to solve only three transport equations (for $\widetilde{Z}, \widetilde{Z^{\prime \prime 2}}$ and $\widetilde{C}$ ) to evaluate all of the thermo-chemical quantities, thus avoiding the expensive solution of one transport equation for each chemical species.

Model $\mathrm{B}$, based on the SMLD approach to model the joint PDF, does not need any assumption about the form of $\widetilde{P}(Z, \Lambda)$. Following such an approach, the probability distribution can be evaluated as a function of an arbitrary number of moments of $Z$ and $\Lambda$. It is noteworthy that, even though equation (1) is based on the assumption that $Z$ and $\Lambda$ are independent, one can properly take into account the statistical correlation between $Z$ and $\Lambda$ employing the SMLD joint-PDF in the evaluation of the effects of turbulence 32 .

In this work, the first two moments of the joint probability density function $\widetilde{P}(\vec{x})$, where $\vec{x}=(Z, \Lambda)^{T}$, are assumed to be known; therefore, the joint-PDF reads [36] :

$$
\begin{aligned}
\widetilde{P}_{S M L, 2}(Z, \Lambda) & =\frac{1}{\mu_{0}} \exp \left\{-\left[\mu_{1,1}(Z-\widetilde{Z})+\mu_{1,2}(\Lambda-\widetilde{\Lambda})\right]\right. \\
& \left.-\frac{1}{2}\left[\mu_{2,11}(Z-\widetilde{Z})^{2}+\mu_{2,12}(Z-\widetilde{Z})(\Lambda-\widetilde{\Lambda})+\mu_{2,21}(\Lambda-\widetilde{\Lambda})(Z-\widetilde{Z})+\mu_{2,22}(\Lambda-\widetilde{\Lambda})^{2}\right]\right\} .
\end{aligned}
$$

In the equation above, $\mu_{0}$ is a scalar, $\overrightarrow{\mu_{1}}$ is a two - component vector, and $\overleftrightarrow{\mu_{2}}$ is a square matrix of rank two:

$$
\begin{aligned}
\mu_{0} & =\int d \vec{x} \widetilde{P}_{S M L, 2}(\vec{x}), \\
-\mu_{1, i} & =\int d \vec{x} \partial_{x_{i}} \widetilde{P}_{S M L, 2}(\vec{x})=\beta\left(1 ; \widetilde{\xi_{i}}, \widetilde{\xi_{i}^{\prime \prime 2}}\right)-\beta\left(0 ; \widetilde{\xi}_{i}, \widetilde{\xi_{i}^{\prime \prime 2}}\right), \\
\delta_{k l}-\mu_{2, k n} \widetilde{\xi_{n}^{\prime} \xi_{l}^{\prime}} & =\int d \vec{x} \partial_{x_{k}}\left(\left(x_{l}-\widetilde{\xi}_{l}\right) \widetilde{P}_{S M L, 2}(\vec{x})\right)=\beta\left(1 ; \widetilde{\xi}_{k}, \widetilde{\xi_{k}^{\prime} \xi_{l}^{\prime}}\right)-\widetilde{\xi}_{k} \mu_{1, l},
\end{aligned}
$$


where $i, k, n$, and $l$ indicate the vector components; $\widetilde{\xi}_{i}, \widetilde{\xi}_{i}^{\prime}$ and $\widetilde{\xi_{i}^{\prime \prime}}$ are the mean $\left(\widetilde{x_{i}}\right)$, the fluctuation $\left(x_{i}-\widetilde{x}_{i}\right)$ and the variance $\widetilde{\left(x_{i}^{\prime \prime 2}\right)}$ of the $i$-th component of $\vec{x}$, respectively; finally, $\beta$ indicates the beta distribution function. In model $\mathrm{B}$, one has to solve four additional transport equations (for $\widetilde{Z}, \widetilde{Z^{\prime \prime 2}}, \widetilde{C}$, and $\widetilde{C^{\prime \prime 2}}$ ) to evaluate all of the thermo-chemical quantities.

\section{Governing equations}

\subsection{Flow equations and numerical solution procedure}

The numerical method developed in 37] has been employed to solve the steady-state RANS equations with $k-\omega$ turbulence closure. For an axisymmetric multi-component reacting compressible flow the system of governing equations can be written as:

$$
\partial_{t} \vec{Q}+\partial_{x}\left(\vec{E}-\vec{E}_{\nu}\right)+\partial_{y}\left(\vec{F}-\vec{F}_{\nu}\right)=\vec{S}
$$

where $t$ is the time variable; $x$ and $y$ are the axial and the radial coordinate, respectively; $\vec{Q}=\left(\bar{\rho}, \bar{\rho} \widetilde{u}_{x}, \bar{\rho} \widetilde{u}_{y}, \bar{\rho} \widetilde{H}-\right.$ $\left.\widetilde{p}_{t}, \bar{\rho} \widetilde{k}, \bar{\rho} \widetilde{\omega}, \bar{\rho} \widetilde{R}_{n}\right)$ is the vector of the conserved variables; $\vec{E}, \vec{F}$, and $\vec{E}_{v}, \vec{F}_{v}$ are the inviscid and viscous flux vectors 41, respectively; $\vec{S}$ is the vector of the source terms; $\bar{\rho},\left(\widetilde{u}_{x}, \widetilde{u}_{y}\right), \widetilde{H}$ indicate the Reynolds-averaged value of density, the Favre-averaged values of velocity components and specific total enthalpy given by $\widetilde{H}=\widetilde{h}+\frac{1}{2}\left(\widetilde{u}_{x}^{2}+\widetilde{u}_{y}^{2}\right)+\frac{5}{3} \widetilde{k}$ with $\widetilde{h}$ accounting for the species enthalpy per unit mass, respectively; $\widetilde{p}_{t}=\widetilde{p}+\frac{2}{3} \widetilde{k}$, $\widetilde{p}$ being the Favre-averaged value of pressure; $\widetilde{k}$ and $\widetilde{\omega}$ are the Favre-averaged values of the turbulence kinetic energy and of its specific dissipation rate; $\widetilde{R}_{n}$ is a generic set of conserved variables related to the combustion model. In this framework, $\widetilde{R}_{n}$ is the set of independent variables of the flamelet model, namely, $\widetilde{Z}, \widetilde{Z^{\prime \prime 2}}, \widetilde{C}$, $\widetilde{C^{\prime \prime 2}}$ (see the following subsection).

The heat flux in the total energy equation, namely,

$$
q=-\rho c_{p} D_{T} \nabla T+\sum_{n=1}^{N_{s}} \rho V_{n} Y_{n} h_{n}+\dot{q}_{\text {react }},
$$

is composed of three terms since the Dufour effect is neglected; $D_{T}$ is the thermal diffusivity, $c_{p}$ the specific heat at constant pressure; the mass diffusion term is modelled by the Fick law considering $V_{n}=-D \frac{\nabla Y_{n}}{Y_{n}}$, assigning mixture diffusivity, $D$, to each species and so assuming unitary Lewis number [22]. The third term at the right hand side of the above equation represents the heat release rate:

$$
\dot{q}_{\text {react }}=\sum_{k=1}^{N} \Delta h_{f, k}^{o} \dot{\omega}_{k}
$$

where $k$ is the species index, $\Delta h_{f, k}^{o}$ is the mass formation enthalpy and $\dot{\omega}_{k}$ is the production rate of species $k$.

\subsection{Turbulent FPV transport equations}

For the case of turbulent flames, the solution of the SLFE, namely equation (2), is expressed in terms of the Favre averages of $Z$ and $C$ and of their variance. Using model A, one can tabulate all chemical quantities in terms of $\widetilde{Z}, \widetilde{Z^{\prime \prime 2}}$ and $\widetilde{C}$, since, due to the properties of the $\delta$-distribution, the model is independent of $\widetilde{C^{\prime \prime 2}}$. On the other hand, model B expresses $\phi$ also in terms of $\widetilde{C^{\prime \prime 2}}$ and therefore an additional transport equation needs to be solved. In this case, the transport equations for the combustion model (included in 
equation (21)) are written as:

$$
\begin{aligned}
\partial_{t}(\bar{\rho} \widetilde{Z})+\vec{\nabla} \cdot(\bar{\rho} \widetilde{\vec{u}} \widetilde{Z}) & =\vec{\nabla} \cdot\left[\left(D+D_{\widetilde{Z}}^{t}\right) \bar{\rho} \vec{\nabla} \widetilde{Z}\right] \\
\partial_{t}\left(\bar{\rho} \widetilde{Z^{\prime \prime 2}}\right)+\vec{\nabla} \cdot\left(\bar{\rho} \widetilde{\vec{u}} \widetilde{Z^{\prime \prime 2}}\right) & =\vec{\nabla} \cdot\left[\left(D+D_{\widetilde{Z^{\prime \prime 2}}}^{t}\right) \bar{\rho} \vec{\nabla} \widetilde{Z^{\prime \prime 2}}\right]-\bar{\rho} \widetilde{\chi}+2 \bar{\rho} D_{\widetilde{Z}}^{t}(\vec{\nabla} \widetilde{Z})^{2}, \\
\partial_{t}(\bar{\rho} \widetilde{C})+\vec{\nabla} \cdot(\bar{\rho} \widetilde{\vec{u}} \widetilde{C}) & =\vec{\nabla} \cdot\left[\left(D+D_{\widetilde{C}}^{t}\right) \bar{\rho} \vec{\nabla} \widetilde{C}\right]+\bar{\rho} \dot{\omega}_{C}, \\
\partial_{t}\left(\bar{\rho} \widetilde{C^{\prime \prime 2}}\right)+\vec{\nabla} \cdot\left(\bar{\rho} \widetilde{\vec{u}} \widetilde{C^{\prime \prime 2}}\right) & =\vec{\nabla} \cdot\left[\left(D+D_{\widetilde{C^{\prime \prime 2}}}^{t}\right) \bar{\rho} \vec{\nabla} \widetilde{C^{\prime \prime 2}}\right]-\bar{\rho} \widetilde{\chi} C+2 \bar{\rho} D_{\widetilde{C}}^{t}(\vec{\nabla} \widetilde{C})^{2}+2 \bar{\rho} \widetilde{C^{\prime \prime}} \dot{\omega}_{C}^{\prime \prime},
\end{aligned}
$$

where $\widetilde{\chi}_{C}$ is modelled in terms of $\widetilde{Z^{\prime \prime 2}}$ and $\widetilde{C^{\prime \prime 2}}$ [33], namely $\widetilde{\chi}_{C}=\frac{\widetilde{Z^{\prime \prime 2}} \chi}{{\widetilde{C^{\prime \prime 2}}}^{2}}, D=\nu / \operatorname{Pr}$ is the diffusion coefficient for all of the species; $\nu$ and $\operatorname{Pr}$ are the kinematic viscosity and the Prandtl number, respectively; $D_{\widetilde{Z}}^{t}=D_{\widetilde{Z^{\prime \prime 2}}}^{t}=D_{\widetilde{C}}^{t}=D_{\widetilde{C^{\prime \prime 2}}}^{t}=\nu_{t} / S c_{t}$ are the turbulent mass diffusion coefficients, $S c_{t}$ being the turbulent Schmidt number equal to 0.8 ; finally, $\dot{\omega}_{C}$ is the source term for the progress variable precomputed and tabulated in the flamelet library. At every iteration, the values of the flamelet variables are updated using equations (24)-(27) and the Favre-averaged thermo-chemical quantities are computed, using equation (8). Such solutions provide the mean mass fractions which are used to evaluate all of the transport properties of the fluid, namely the molecular viscosity, the thermal conductivity and the species diffusion coefficients.

The evaluation of the flamelet library has been performed using the detailed kinetic scheme proposed by Saxena and Williams [42: 244 sub-reactions upon 50 species. The flamelet library has been computed over a grid with 250 uniformly distributed points in the $\widetilde{Z}$ and $\widetilde{C}$ directions, and 50 uniformly distributed points in the $\widetilde{Z^{\prime \prime 2}}$ and $\widetilde{C^{\prime \prime 2}}$ directions and a quadri-linear interpolation scheme is used. The flamelet library has been evaluated considering a constant background pressure equal to $100 \mathrm{kPa}$ and boundary conditions for $Z$ and $C$ corresponding to the conditions of the air and $H_{2}$ streams. Indeed, the air stream is represented as $\widetilde{Z}=0$ and $\widetilde{C}=0$, while the fuel jet is given by $\widetilde{Z}=1$ and $\widetilde{C}=0$. The solution of the SLFE has been obtained by using the FlameMaster code [43].

\subsection{Numerical scheme and boundary conditions}

A cell-centred finite volume space discretization is used on a multi-block structured mesh. The convective and viscous terms are discretized by the third-order-accurate Steger and Warming [44] flux-vector-splitting scheme and by second-order-accurate central differences, respectively. An implicit time marching procedure is used with a factorization based on the diagonalization procedure of Pulliamm and Chaussee [45], employing a scalar alternating direction implicit (ADI) solution procedure [46. Steady flows are considered and the ADI scheme is iterated in the pseudo-time until a residual drop of at least five orders of magnitude for all of the conservation-law equations (21) is achieved. Characteristic boundary conditions for the flow variables are imposed at inflow and outflow points. In particular, a plug flow is imposed at the inlet points of the computational domain so as to match the experimental conditions at the inlet section of the chamber, see table 1. Moreover, $k, \omega$, and $\tilde{R}_{n}$ are assigned at inflow points, whereas they are linearly extrapolated at outflow points. Finally, no slip and adiabatic conditions are imposed at walls, where $k$ is set to zero and $\omega$ is evaluated as proposed in [47:

$$
\omega=60 \frac{\nu}{0.09 y_{n, 1}^{2}}
$$

where $y_{n, 1}$ is the distance of the first cell center from the wall; the homogeneous Neumann boundary condition is used for $\tilde{R}_{n}$ (non-catalytic wall). Finally, symmetry conditions are imposed at the axis.

\section{Results and discussion}

\subsection{Description of the test case}

The hydrogen-air supersonic combustion burner studied by Cheng et al. 48, at the NASA Langley Research Center has been considered as a suitable test case for the proposed method. At the inlet section 


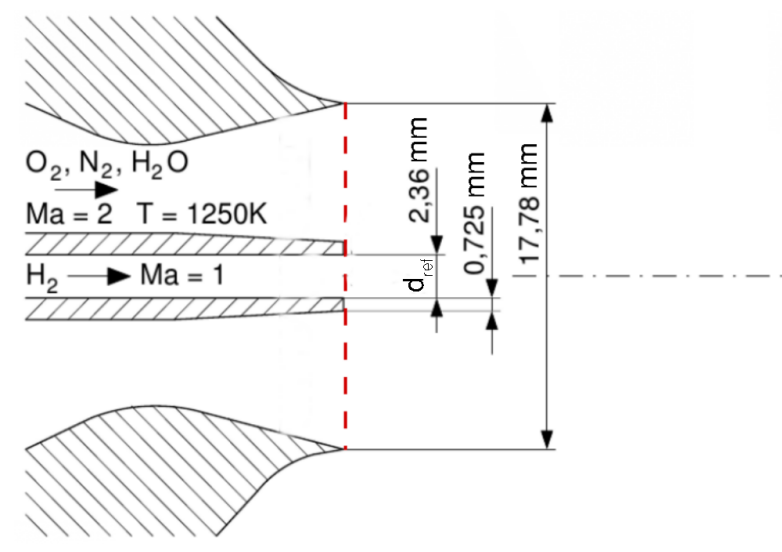

Figure 1: Schematic of the Cheng's Flame burner. The red-dashed line represents the exit section of the burner and so the inlet section of the chamber.

of the chamber, the supersonic flow (see figure 1) is characterized by an annular axisymmetric jet of hot vitiated wet air at Mach number equal to 2 , average axial velocity of $1420 \mathrm{~m} / \mathrm{s}$, temperature of $1250 \mathrm{~K}$ and pressure of $107 k P a$. The values of the inner and outer diameters of the vitiated air annular duct are equal to $3.812 \mathrm{~mm}$ and $17.78 \mathrm{~mm}$, respectively. The composition of the vitiated air is: $Y_{\mathrm{O}_{2}}=0.201$, $Y_{\mathrm{H}_{2} \mathrm{O}}=0.255$ and $Y_{\mathrm{N}_{2}}=0.544$, produced by a pre-combustion at low temperature 48. The diameter of the fuel stream is $d_{\text {ref }}=2.362 \mathrm{~mm}$, taken as the reference length. The hydrogen flow is estimated to be chocked with average axial velocity of $1780 \mathrm{~m} / \mathrm{s}$, temperature of $545 \mathrm{~K}$, and pressure of $112 \mathrm{kPa}$. The operating conditions reported by Cheng et al. [48, are summarized in table 1 together with the turbulent intensity levels, $T u_{i n}$, and the turbulent viscosity, $\nu_{T_{i n}}$. The computational domain, shown in figure 2 , is axisymmetric and includes the divergent part of the air nozzle, necessary to recover the correct flow quantities at the inlet section of the chamber; it extends $150 d_{r e f}$ and $50 d_{\text {ref }}$ along the axial and radial directions, respectively, and, after a grid refinement study, has been discretized using about 100000 cells. The characteristic cell lengths at the exit of the divergent part of the nozzle are about $0.13 \mathrm{~mm}$ and $0.05 \mathrm{~mm}$ in the axial and radial directions, respectively. Numerical results obtained using the two models described in the previous sections are discussed and validated versus the experimental data of Cheng et al. [48, and the numerical results of [7, 49. In particular, a detailed analysis of the flame structure is provided.

\subsection{Comparison between numerical and experimental data}

Table 1: Parameters for the simulation of the Cheng's combustion chamber 48].

\begin{tabular}{l|cccccccccc}
\hline \multirow{3}{*}{$H_{2}$ jet } & $M a$ & $u(\mathrm{~m} / \mathrm{s})$ & $T(\mathrm{~K})$ & $p(\mathrm{kPa})$ & $Y_{\mathrm{H}_{2}}$ & $Y_{\mathrm{O}_{2}}$ & $Y_{\mathrm{H}_{2} \mathrm{O}}$ & $Y_{\mathrm{N}_{2}}$ & $T u_{i n}$ & $\nu_{T_{i n}}\left(\mathrm{~m}^{2} / s\right)$ \\
& 1 & 1780 & 545 & 112 & 1 & 0 & 0 & 0 & $5.78 \%$ & 0.00023 \\
Air stream & 2 & 1420 & 1250 & 107 & 0 & 0.201 & 0.255 & 0.544 & $10.24 \%$ & 0.00034 \\
\hline
\end{tabular}

Figure 3 provides the Mach number contours obtained using model A and model B. The close-up view of the near-burner region (bottom-left and -right panels) indicates that the inlet Mach number value is very close to the experimental data for both computations. The distributions of the streamwise component of the velocity, $u$, at several abscissae along the chamber are shown in figure 4 A good agreement with the experimental data [4] is obtained for both models. Figure 5 (top frame) shows a qualitative comparison between the temperature contours evaluated with the two models. It appears that the computed flame shapes are quite different. Model A predicts a reaction zone attached to the burner, whereas model B correctly predicts the flame detachment. In the bottom frame of figure 5 one can find the two corresponding 

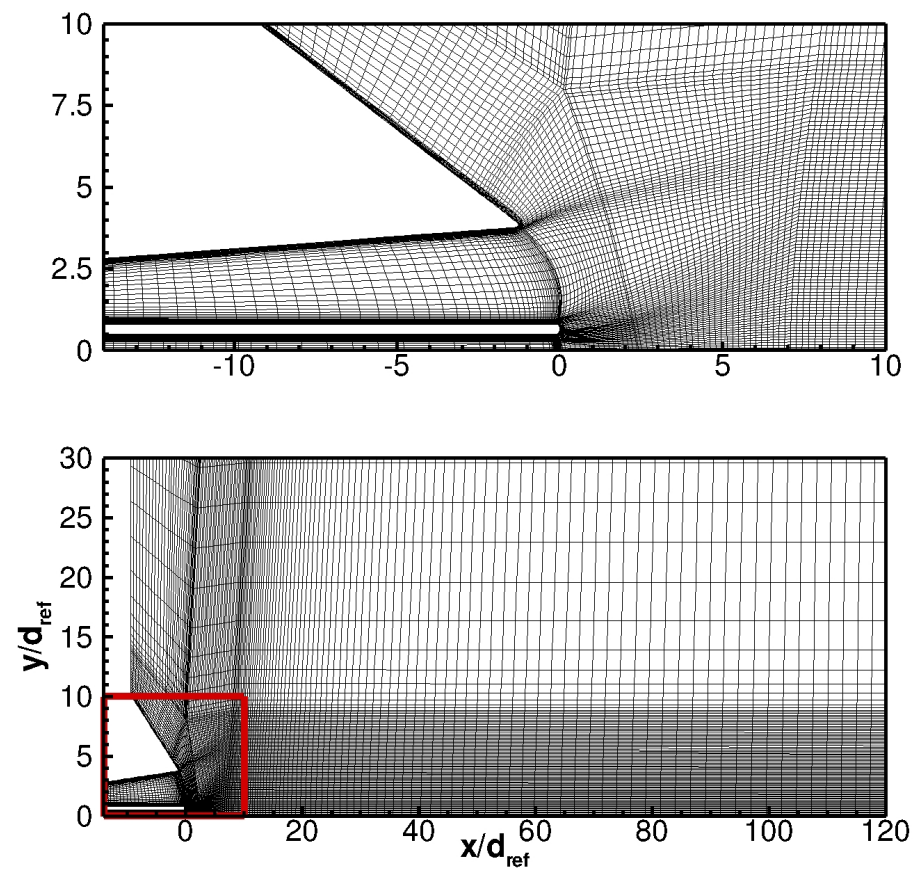

Figure 2: Computational domain discretization (lower frame) and a detail of the injectors (upper frame).
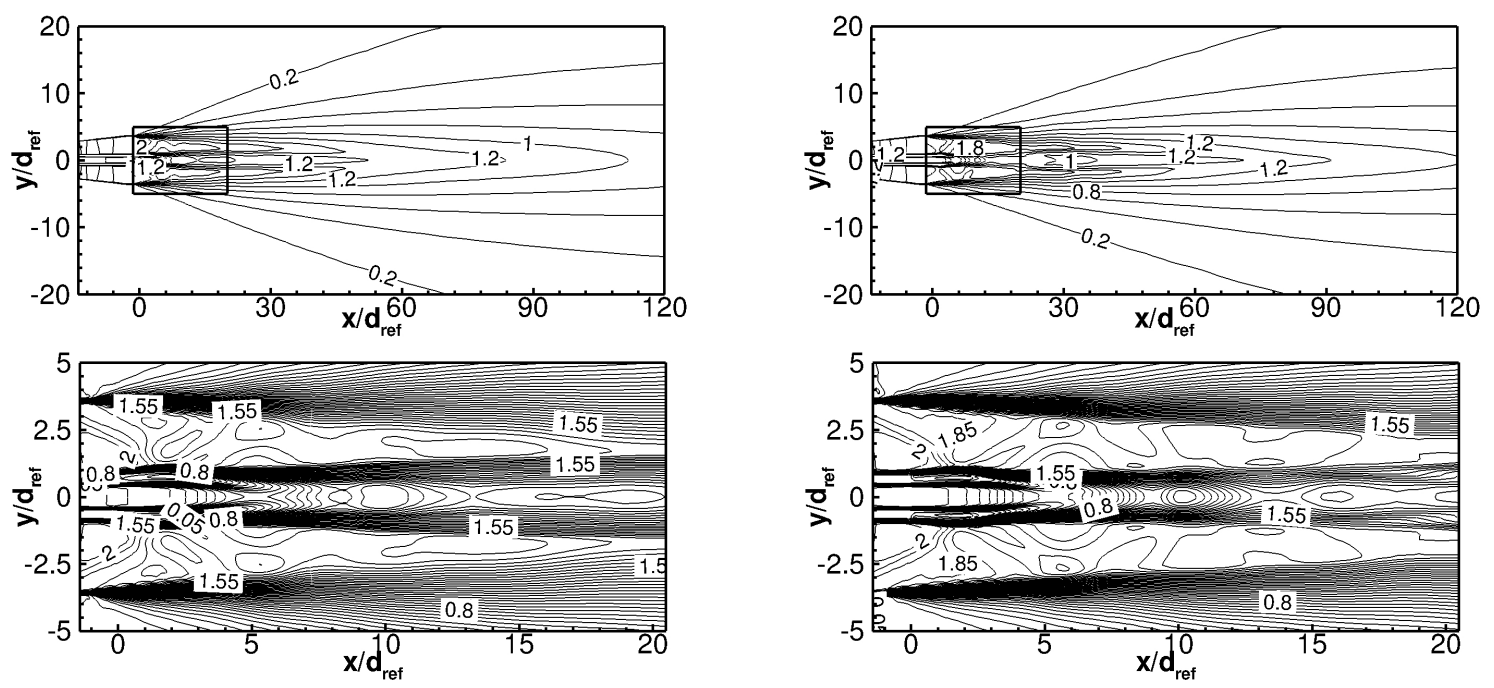

(a) Model A

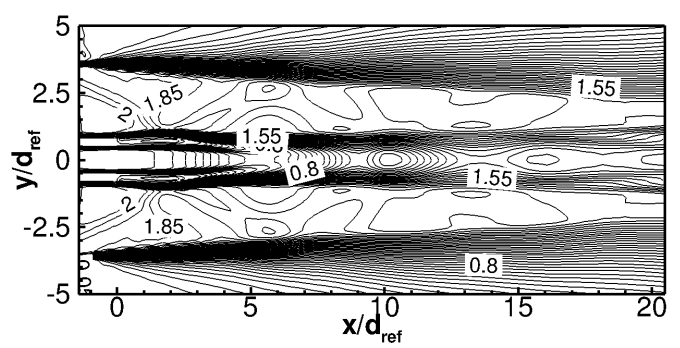

(b) Model B

Figure 3: Mach number contours obtained with model A (left) and model B (right), with a close up of the near-burner region (bottom). 

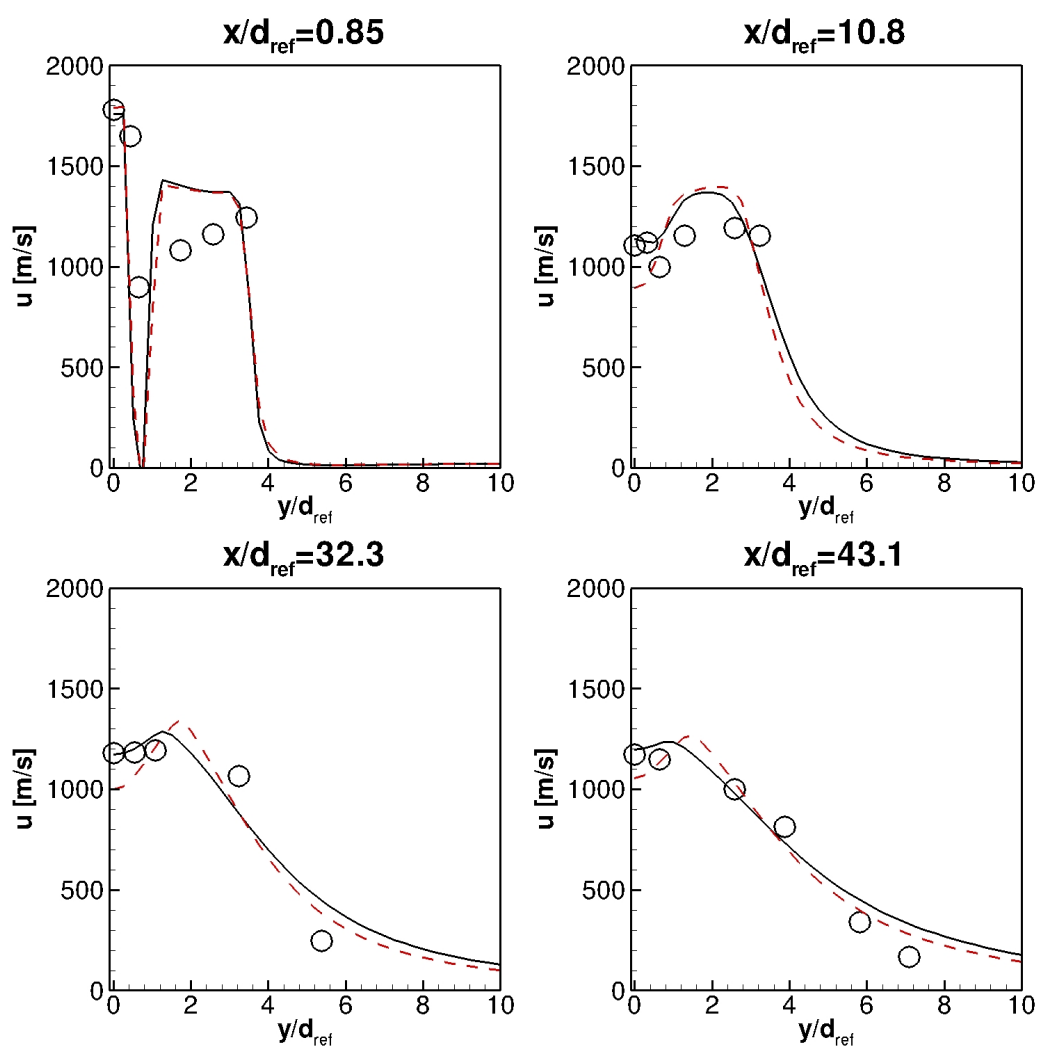

Figure 4: Stream-wise velocity distributions at several radial sections: model A, red dashed line; model B, black solid line; symbols, experimental data. 


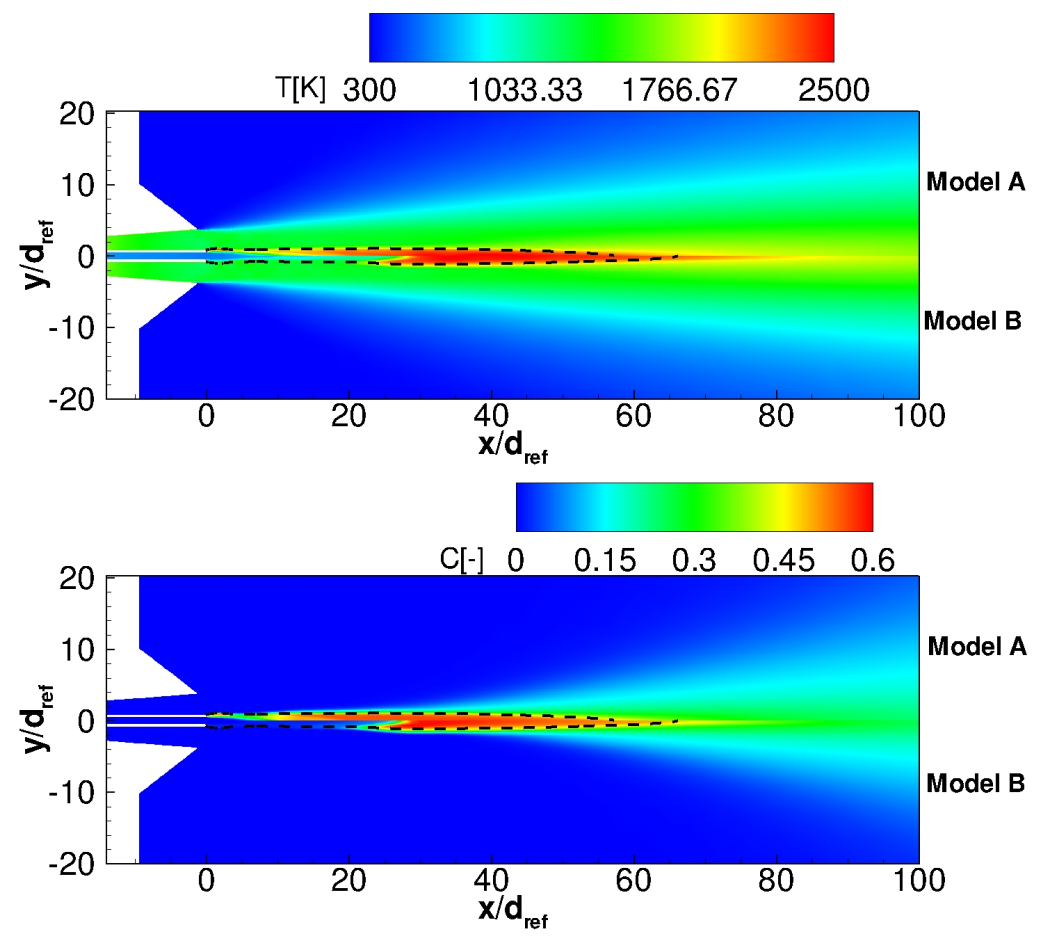

Figure 5: Temperature (top) and progress variable (bottom) contours for the Cheng's combustion chamber with the $Z_{\text {st }}$ isoline superimposed (black dashed line).

progress-variable contours. The solution obtained using model A provides non-zero values for the progress variable in the region close to the burner, indicating that the reaction is active. Figure 6 presents the temperature field obtained using model A (left panel) and model B (right panel) in comparison with the results of an accurate LES by Boivin et al. 77. One can observe that model B predicts the lift-off height, evaluated as the position of maximum temperature gradient, at about $x=26 d_{r e f}$, whereas model A predicts an height of about $9 d_{\text {ref }}$. The results by Boivin et al. 77, used as reference, predicts the stabilization at $25 d_{\text {ref }}$. For both models, the jet shape and the aperture of the flame at $x / d_{\text {ref }}=50$, equal about to $10 d_{\text {ref }}$, are in good agreement with the experimental data (not shown). For a quantitative analysis of the results, figures $7 \sqrt{9}$ provide the comparison among the results obtained using the two FPV models, the numerical results provided in [7, 49, and the experimental data [48. Figure 7 shows the distribution of the main thermo-chemical quantities along the axis of the burner. The evaluation of the $\widetilde{Z}$ and $\widetilde{Z^{\prime \prime 2}}$ are very slightly improved by model B; a reasonable improvement of the prediction of the distribution of $O H$ mass fraction is also obtained; whereas, $\mathrm{H}_{2}$ and $\mathrm{H}_{2} \mathrm{O}$ mass fractions are quite well predicted in comparison with model A. It appears that the flame core is not well reproduced using model $\mathrm{A}$, which indeed provides a too high reaction rate, so that the reactions may occur wherever the two flows $\left(H_{2}\right.$-wet-air $)$ are mixing. As a consequence, model A predicts $Y_{\mathrm{H}_{2} \mathrm{O}}$ maximum at about $10 x / d_{\text {ref }}$, as shown in the middle right frame of figure 7 . On the other hand, model $\mathrm{B}$ can reproduce the ignition spatial delay with a strong increase of the temperature at about $x / d_{\text {ref }}=30$ (figure 7 middle-left frame).

Figure 7 also provides the numerical results obtained with an LES by Boivin et al. [7, for comparison; they appear to be in good agreement with the results obtained by model B, confirming the good level of accuracy achieved by such a model. Analysing the temperature and water mass fraction experimental distributions shown in figure 7 . one can notice that the temperature begins to decrease at $x / d_{\text {ref }}=50$ whereas the water mass fraction increases up to about $x / d_{\text {ref }}=65$. This is due to the entrainment of the surrounding unburnt-air into the flame, which induces an increase of the mass flow rate and allows the 


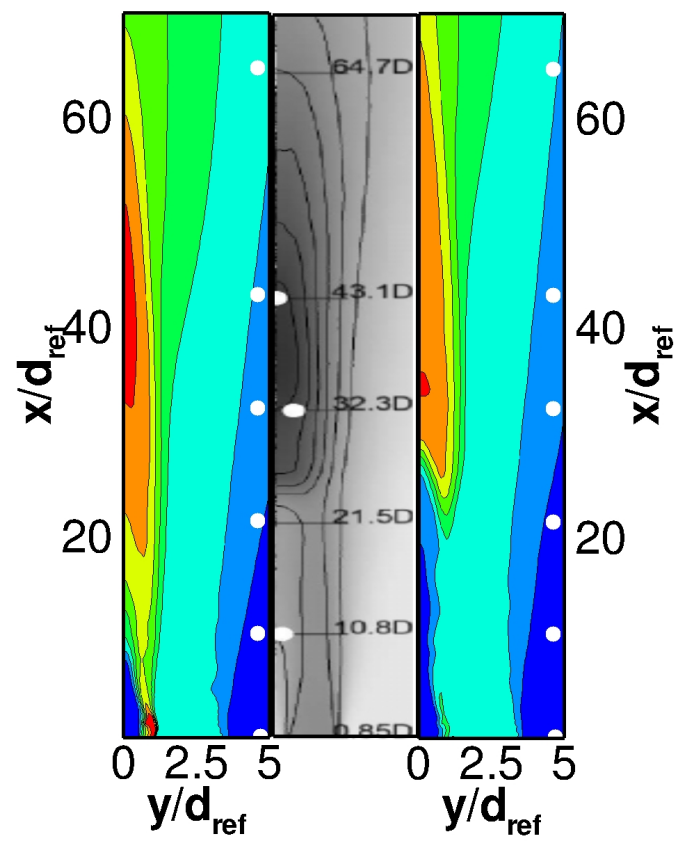

Figure 6: Comparison between the temperature contours computed by model A (left), Boivin et al. [7] (center), model B (right). The contour lines are plotted from $1000 \mathrm{~K}$ to $2500 \mathrm{~K}$ with step equal to $250 \mathrm{~K}$.

reactions to occur even if the flame cannot heat up the surrounding flow. In particular, the early increase of the temperature and of the water mass fraction, obtained by model A, indicate that the heat is released very close to the burner. As a consequence, the entrainment of the surrounding unburnt-air into the flame is strongly overestimated using such a model with respect to model B. The entrainment can be evaluated by computing the total mass flow rate through the inlet of the chamber, at $x / d_{r e f}=0$, and at the sections $x / d_{\text {ref }}=25$ and $x / d_{\text {ref }}=50$. Due to the axial-symmetry, the effective surfaces intersected by the flow can be estimated as the circles with radius equal to the maximum extension of the temperature profiles, namely $\left(y / d_{\text {ref }}\right)_{0}=5.540,\left(y / d_{\text {ref }}\right)_{25}=16.02$, and $\left(y / d_{\text {ref }}\right)_{50}=27.115$ (see figures 8 ). The mass fluxes computed using the solution of model B are equal to $\dot{m}_{x / d_{\text {ref }}=0}=96.28 \mathrm{~g} / \mathrm{s}, \dot{m}_{x / d_{\text {ref }}=25}=511.78 \mathrm{~g} / \mathrm{s}$, and $\dot{m}_{x / d_{r e f}=50}=1163.77 \mathrm{~g} / \mathrm{s}$, respectively. The different behaviour of model A reflects in the evaluation of the mass fluxes which are: $\dot{m}_{x / d_{r e f}=0}=98.07 \mathrm{~g} / \mathrm{s}, \dot{m}_{x / d_{r e f}=25}=681.83 \mathrm{~g} / \mathrm{s}$, and $\dot{m}_{x / d_{r e f}=50}=1202.08 \mathrm{~g} / \mathrm{s}$.

Figure 9 shows the distribution of some relevant thermo-chemical variables at several abscissae along the chamber axis, comparing the results obtained by the two FPV models, an LES with reduced chemistry evaluation [7], a mixture-fraction based model [49], and experimental results [4]. The $\mathrm{H}_{2}$ and $\mathrm{O}_{2}$ mass fraction distributions agree fairly well with LES and experimental data. The improvement with respect to the results of model $\mathrm{A}$ is more evident in the near axis region. Model A predicts a thin reacting zone near the burner, so that in sections at $x / d_{\text {ref }}=0.85$ (see figure 8) and $x / d_{\text {ref }}=10.8$ (see figure 9) the temperature distributions present a spike close to the axis. This spike characterizes also the water mass fraction distribution of the mixture-fraction-based model by Thibault and Boivin [49. On the other hand, model $\mathrm{B}$ is not affected by this problem and correctly predicts the flame core.

\subsection{The flame structure}

In this section the structure of the flame is studied with reference to the work of Moule et al. [50] and of Boivin et al. 7] who have analyzed the considered supersonic burner. Moule et al. [50] identify three 

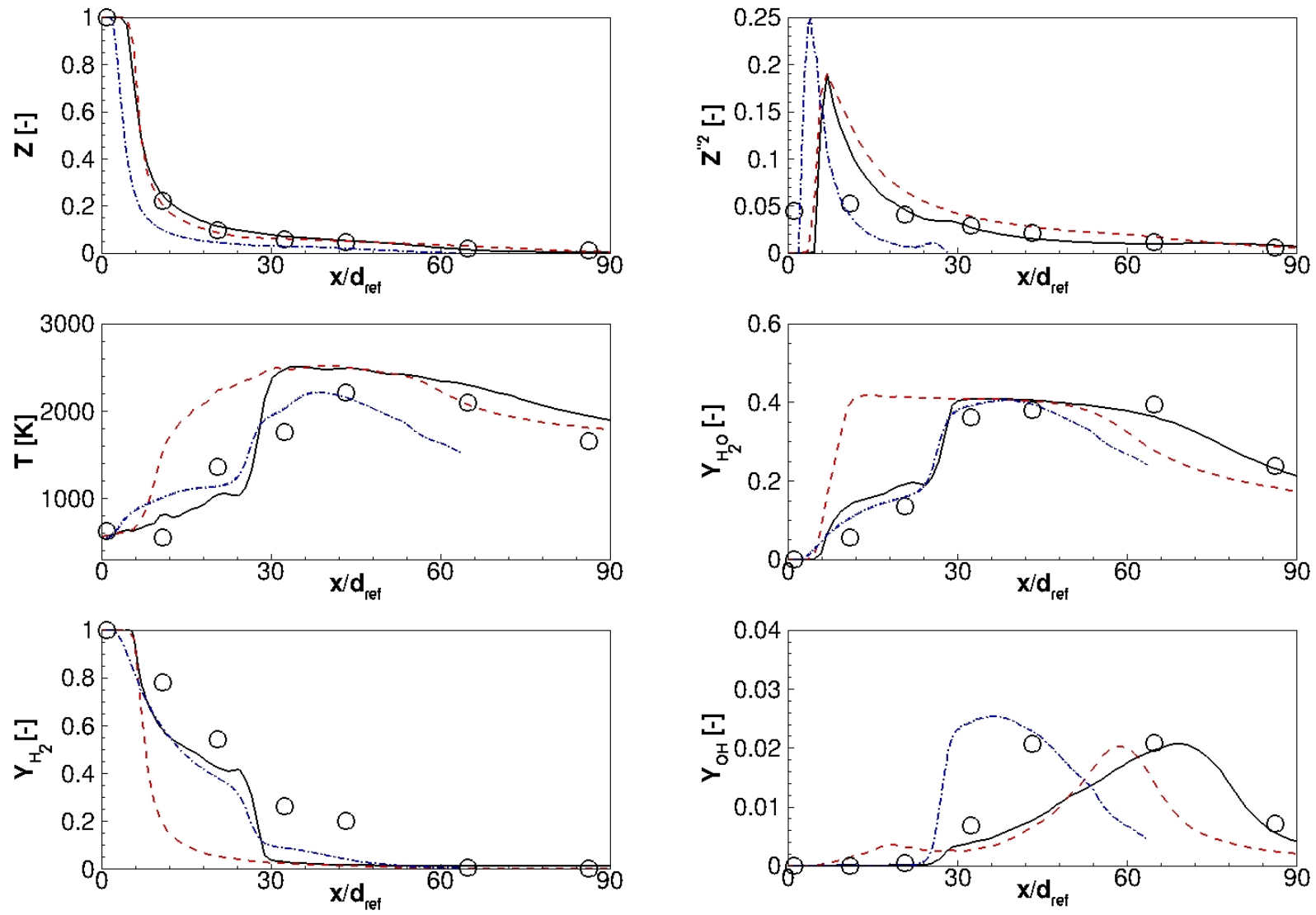

Figure 7: Distributions of different thermo-chemical quantities

along the axis $\left(y / d_{\text {ref }}=0\right)$. Model $\mathrm{A}$, red dashed line; model B, solid black line; Boivin et al. dashed-dotted blue line [7]; symbols, experimental data [48].
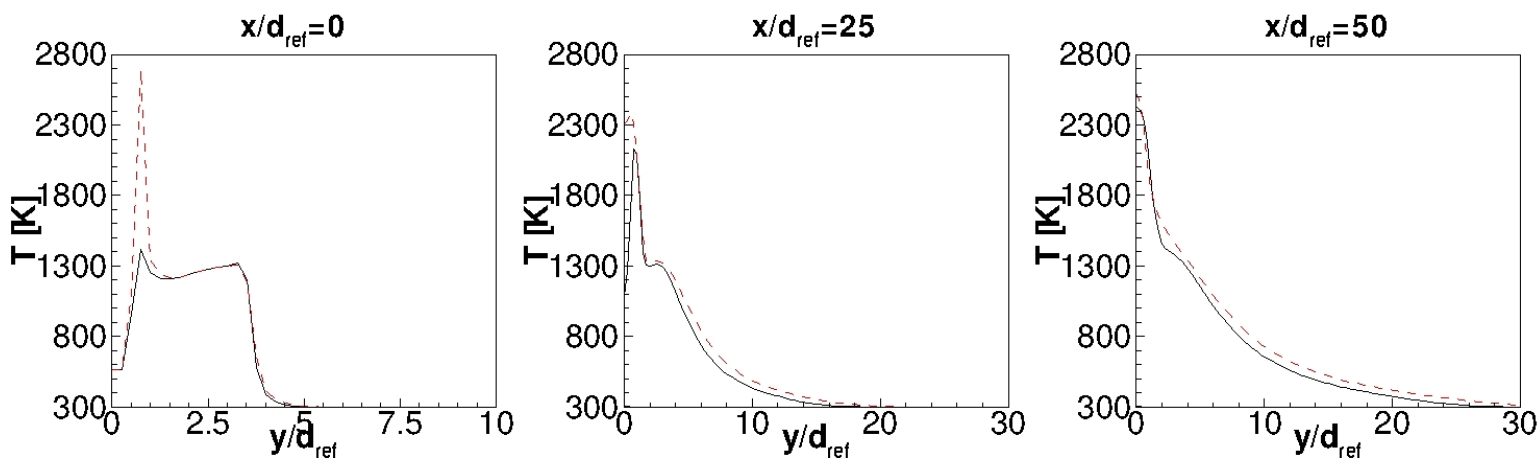

Figure 8: Temperature distributions at $x / d_{r e f}=0, x / d_{r e f}=25$, and $x / d_{r e f}=50$ : model A, red dashed line; model B, solid black line. 

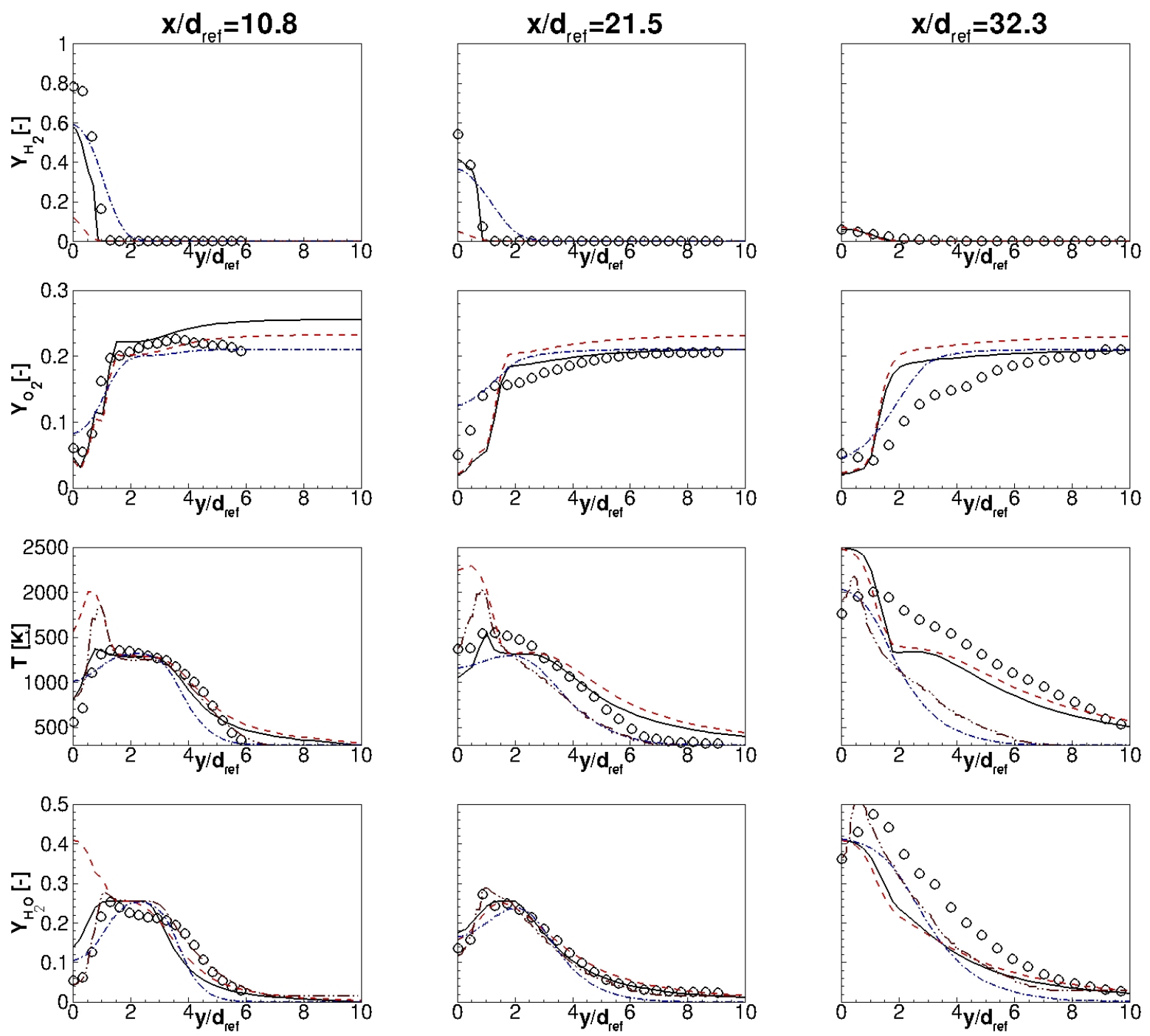

Figure 9: Chemical species distributions at $x / d_{\text {ref }}=10.8, x / d_{\text {ref }}=21.5$, and $x / d_{\text {ref }}=32.3$ : model A, red dashed line; model B, solid black line; Boivin et al. dashed-dotted blue line [7]; Thibault and Boivin dashed-dotted-dotted brown line [49]; symbols, experimental data [4]. 


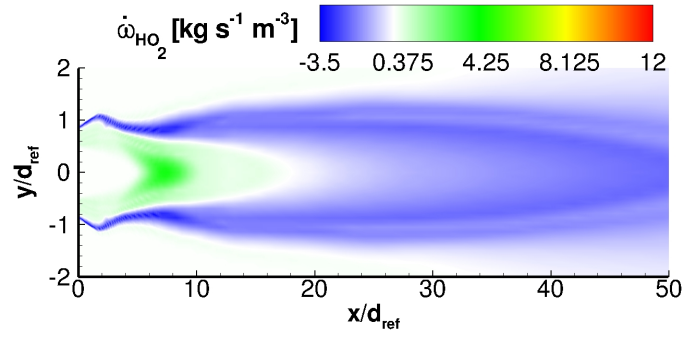

(a) Model A

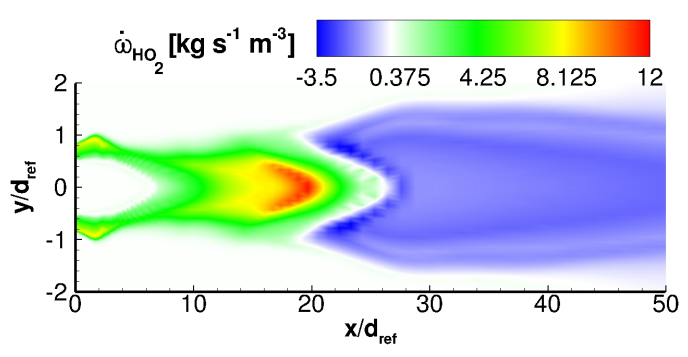

(b) Model B

Figure 10: Model A and Model B solutions: contours of $\dot{\omega}_{\mathrm{HO}_{2}}$.

characteristic regions of the flame, namely: 1$)$ the auto-ignition zone, $10 \leq x / d_{\text {ref }} \leq 18$, where the mixture prepares to ignite; 2 ) the stabilization region, $18 \leq x / d_{\text {ref }} \leq 26$, where the flame starts; 3 ) the combustion region, $30 \leq x / d_{\text {ref }} \leq 40$.

In the first zone, the initial steps of the hydrogen oxidation take place and the reaction produces simple hydrogen radicals. In particular, for hydrogen combustion, the hydroperoxyl radical $\mathrm{HO}_{2}$ can be considered as a good marker of autoignition. However, since the $\mathrm{HO}_{2}$ concentration also increases in fuel-rich reaction zones of ignited mixtures, Boivin et al. 7] proposed a more accurate criterion for detecting autoignition. Such a criterion requires the simultaneous presence of high values of the normalized production rate of $\mathrm{HO}_{2}$ and of the reactivity of the mixture, $\lambda$, defined as the positive eigenvalue of the Jacobian of the chemical source term associated to the autoignition chain-branching reaction [7. For the present supersonic liftoff flame, the results of the LESs discussed in references [50] and [7] confirm the effectiveness of such a criterion in identifying the autoignition region as well as the following transition from the autoignition to the stabilization characterized by the $\mathrm{HO}_{2}$ depletion.

Analysing the results of the present RANS simulations, we can show that model B can provide a flame structure corresponding to the above scenario. Figure 10 shows the contours of the $\mathrm{HO}_{2}$ production rate, $\dot{\omega}_{\mathrm{HO} 2}$, computed using model A (left) and model B (right). This figure indicates that both models predict a substantial $\mathrm{HO}_{2}$ production downstream of the jet exit, in agreement with the results of the LES by Moule et al. 50] (see figure 17 of their paper). Moreover, it appears that the production region is followed by depletion taking place in the stabilization region. Figure 11 shows the contour plot of $\lambda$ together with the $\mathrm{YHO}_{2}=$ $3 \times 10^{-5}$ isoline, considered as the reference value for the high temperature hydrogen autoignition [50]. It appears that the most reactive part of mixture is in the near-burner zone, where the mixing among the reactants is stronger. Both models properly evaluate the reactivity, in good agreement with the results of the LES of 50 (see figure 19 therein). Looking at the contours of $\dot{\omega}_{\mathrm{HO} 2}$ in figure 10 and at the contours of $\lambda$ in figure 11. we can verify the conjecture of Boivin et al. concerning the autoignition region. In fact, we can see that model B predicts the autoignition at $7 \leq x / d_{r e f} \leq 23$ where sufficiently high values of the reactivity and of the $\mathrm{HO}_{2}$ production are achieved. On the other hand, model A predicts a too fast reaction rate, providing a shorter autoignition region for $3 \leq x / d_{\text {ref }} \leq 11$.

In the second zone, the flame finds its stable position and, as shown in figure 12 , it is characterized by high values of the heat release rate. Figure 12 also shows the sonic isoline and the axial temperature profile superposed to the contours of the heat release rate. According to the LES results of [50, although the highest temperature occurs in the subsonic region, $32 \leq x / d_{\text {ref }} \leq 38$, high values of $\dot{q}_{\text {react }}$ are concentrated in the neighbourhood of the stabilization region of the flame, $18 \leq x / d_{r e f} \leq 26$. For the present RANS computations, model A predicts high values of the temperature and of the heat release too close to the burner, whereas, model B provides results in very good agreement with the LES concerning both the heat release region and the temperature profile along the axis of the burner.

As proposed by Boivin et al. [7, it is also possible to employ a unique parameter that identifies the 


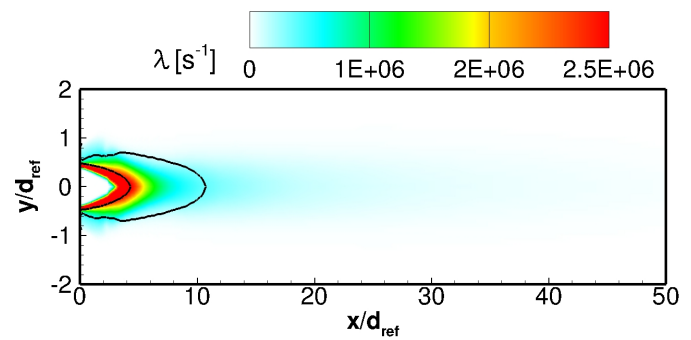

(a) Model A

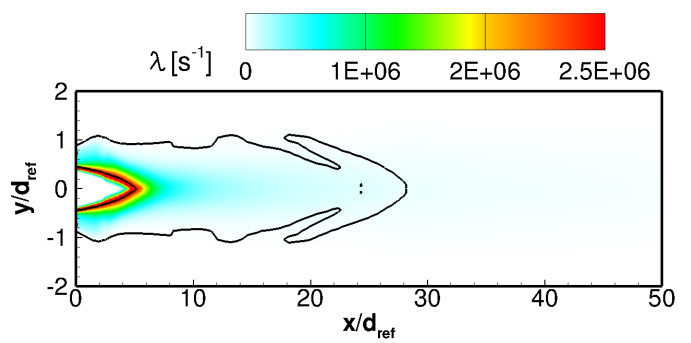

(b) Model B

Figure 11: Model A and Model B solutions: reactivity contour plot with the $Y_{\mathrm{HO}_{2}}$ isoline $\left[3 \times 10^{-5}\right]$ superimposed.

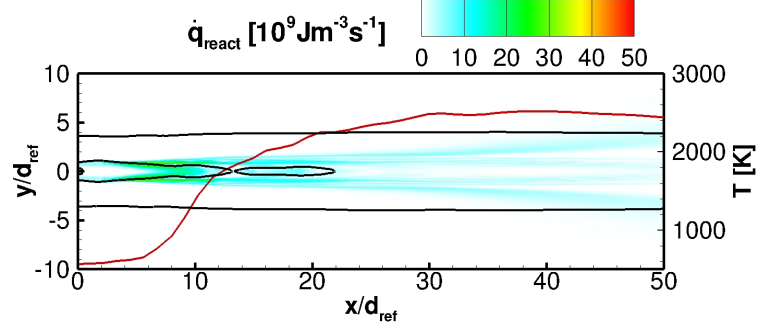

(a) Model A

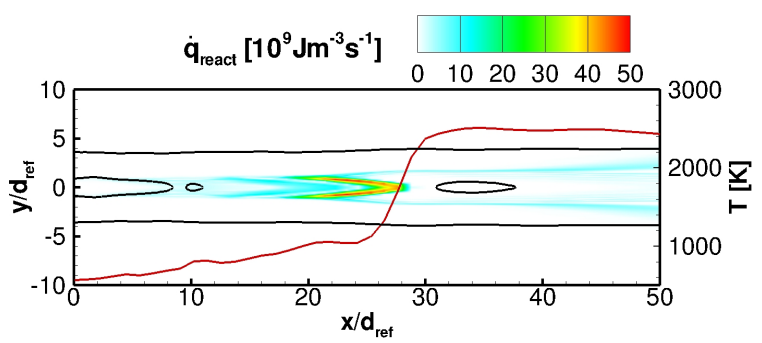

(b) Model B

Figure 12: Model A and Model B solutions: heat reaction rate contour plot with the $M a=1$ isoline (black solid line) and the axial temperature profile (red solid line) superimposed. 


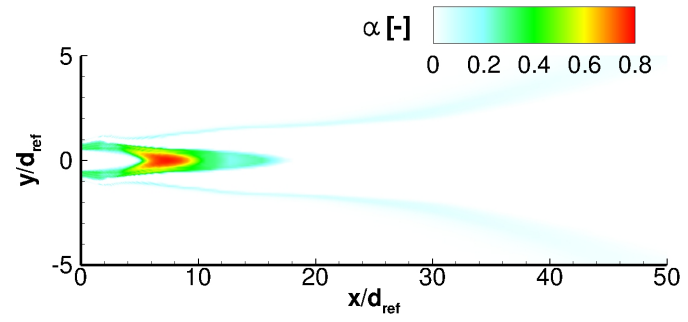

(a) Model A

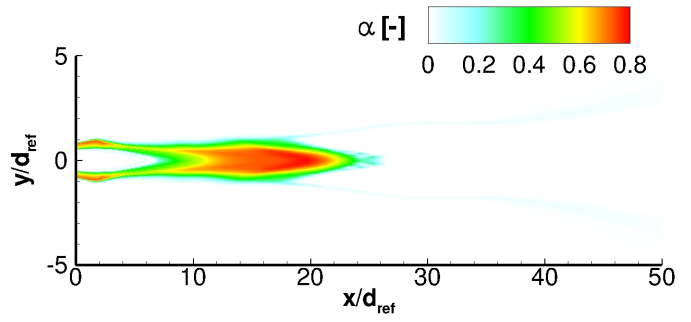

(b) Model B

Figure 13: Model A and Model B solutions: contour plot of $\alpha$.

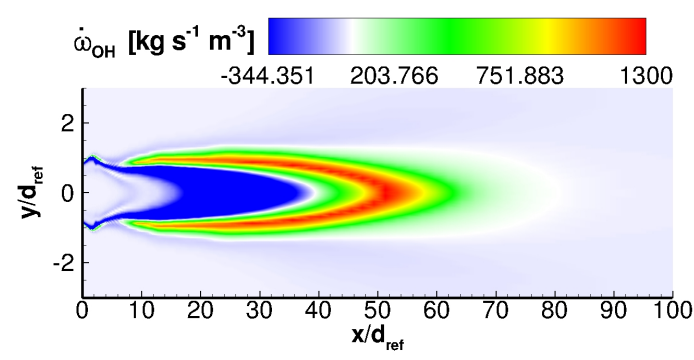

(a) Model A

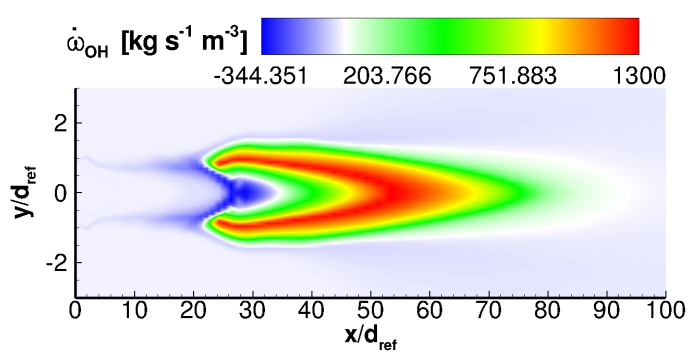

(b) Model B

Figure 14: Model A and Model B solutions: contour plot of $\dot{\omega}_{O H}$.

occurrence of autoignition,

$$
\alpha=\frac{\dot{\omega}_{H O_{2}}^{+}-\dot{\omega}_{H O_{2}}^{-}}{\dot{\omega}_{H O_{2}}^{+}},
$$

with $\dot{\omega}_{\mathrm{HO}_{2}}^{+/-}$the positive/negative part of $\dot{\omega}_{\mathrm{HO}_{2}}$ representing the production and the destruction rates of $\mathrm{HO}_{2}$. According to Boivin et al. [7, the autoignition occurs when $\alpha$ decreases from its maximum reference value $\left(\alpha_{\max } \approx 0.95\right)$ to its minimum reference value $\left(\alpha_{\min } \approx 0.05\right)$. Figure 13 shows the two $\alpha$ contour plots obtained by model $\mathrm{A}$ and $\mathrm{B}$, respectively, confirming that model $\mathrm{B}$ is capable of predicting the transition from the autoignition to the stabilization region at about $27 d_{r e f}$, providing a good estimate of the lift-off height.

Finally, figure 14 shows that in the third region $\left(30 \leq x / d_{\text {ref }} \leq 40\right)$ the combustion develops and is characterized by high values of $O H$ production.

\section{4. $Z$ and $\Lambda$ distributions}

In this section, we focus on the analysis of those flow regions where large differences between the predictions of model $\mathrm{A}$ and $\mathrm{B}$ are observed. In particular, we compare the $\widetilde{P}_{S M L, 2}$ with the $\beta$-distribution for $\mathrm{Z}$ and with the $\delta$-function for $\Lambda$. All the distributions are evaluated using the values of the mean and variance computed by model $\mathrm{B}$ at three points: two of them are on the axis $\left(y / d_{r e f}=0\right)$ and correspond to the lift-off heights evaluated with the two models, $x / d_{r e f}=8$ and $x / d_{r e f}=27$, respectively; the third point is close to the jet exit $\left(x / d_{r e f}=0.85, y / d_{r e f}=0.8\right)$, where a spurious temperature peak is predicted by model A (see figures 8 ).

The first point $\left(x / d_{\text {ref }}=8\right)$ is in the stabilization region for the solution obtained by model $\mathrm{A}$ and falls in the mixing non-burning region for the solution obtained by model B. This is clearly indicated by the 
$\widetilde{Z}=0.34, \widetilde{Z^{\prime \prime 2}}=0.024, \widetilde{\Lambda}=0.008, \widetilde{\Lambda^{\prime \prime 2}}=0.0$

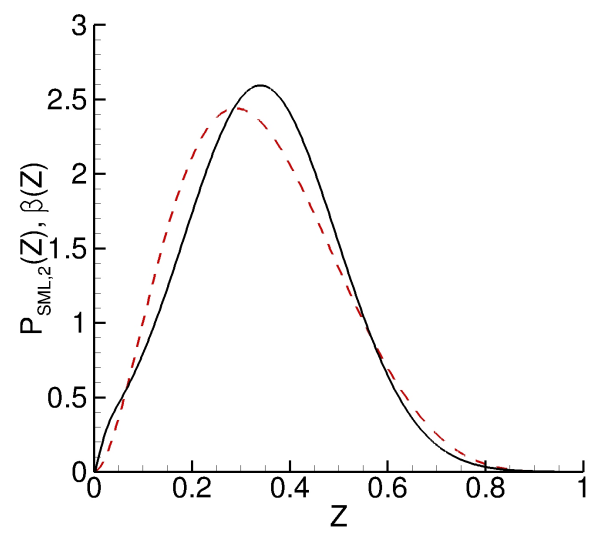

Figure 15: PDFs of $Z$ at the point $\left(x / d_{r e f}, y / d_{r e f}\right)=(8,0)$ using the values obtained by model B: $P_{S M L, 2}(Z)$ (black solid line) and $\beta(Z)$ (red dashed line).
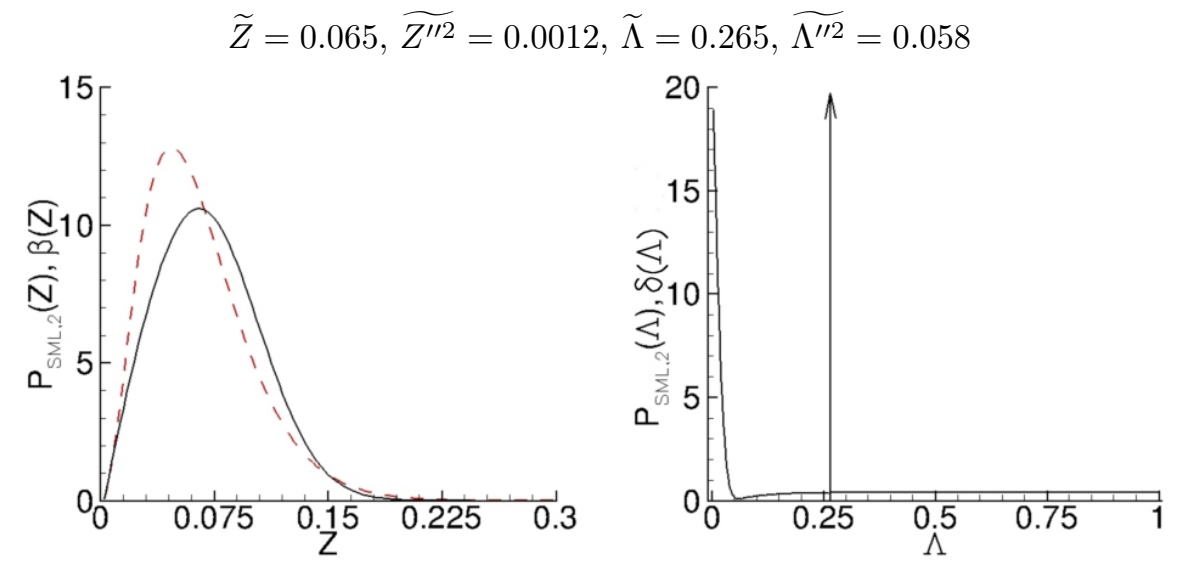

Figure 16: PDFs of $Z$ and $\Lambda$ at the point $\left(x / d_{\text {ref }}, y / d_{r e f}\right)=(27,0)$ using the values obtained by model B. Left panel: $P_{S M L, 2}(Z)$ (black solid line) and $\beta(Z)$ (red dashed line). Right panel: $P_{S M L, 2}(\Lambda)$ (black solid line) and $\delta(\Lambda)$. 

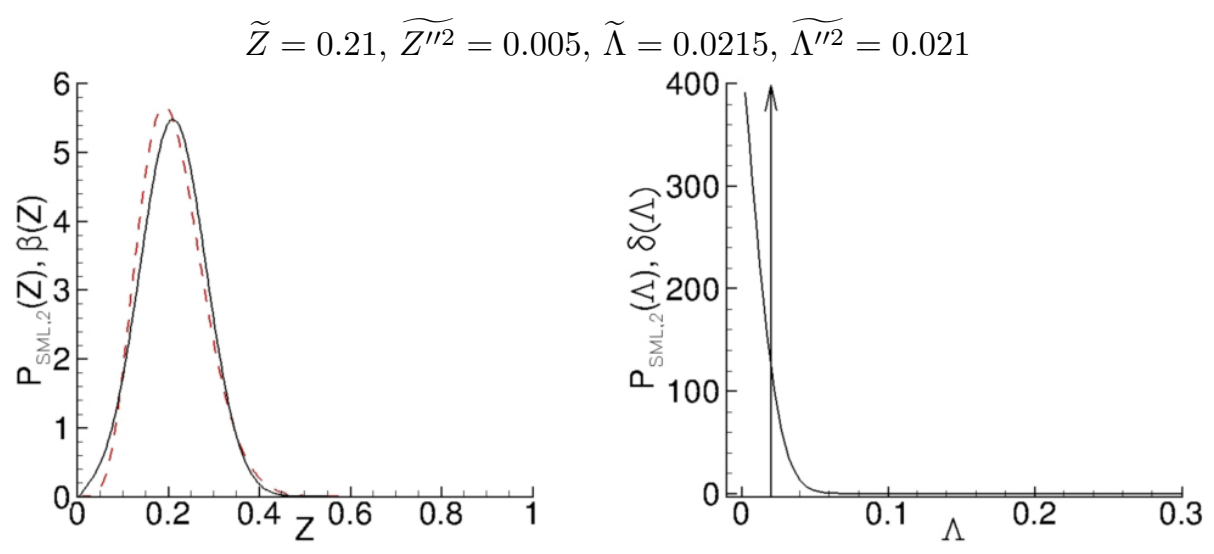

Figure 17: PDFs of $Z$ and $\Lambda$ at the point $\left(x / d_{r e f}, y / d_{r e f}\right)=(0.85,0.8)$ using the values obtained by model B. Left panel: $P_{S M L, 2}(Z)$ (black solid line) and $\beta(Z)$ (red dashed line). Right panel: $P_{S M L, 2}(\Lambda)$ (black solid line) and $\delta(\Lambda)$.

\begin{tabular}{ll|llll|}
\multicolumn{6}{c}{ Table 2: Mean and variance of $Z$ and $\Lambda$ at three selected points. } \\
& & $\widetilde{Z}$ & $\widetilde{Z^{\prime \prime 2}}$ & $\widetilde{\Lambda}$ & \multicolumn{1}{c}{$\widetilde{\Lambda^{\prime \prime 2}}$} \\
\hline \multirow{2}{*}{$\left(x / d_{\text {ref }}, y / d_{\text {ref }}\right)=(8,0)$} & Model A & 0.37 & 0.030 & 0.22 & - \\
& Model B & 0.34 & 0.024 & 0.008 & 0 \\
\hline \multirow{2}{*}{$\left(x / d_{\text {ref }}, y / d_{\text {ref }}\right)=(27,0)$} & Model A & 0.085 & 0.0022 & 0.56 & - \\
& Model B & 0.065 & 0.0012 & 0.265 & 0.058 \\
\hline \multirow{2}{*}{$\left(x / d_{\text {ref }}, y / d_{\text {ref }}\right)=(0.85,0.8)$} & Model A & 0.22 & 0.007 & 0.49 & - \\
& Model B & 0.21 & 0.005 & 0.0215 & 0.021 \\
\hline
\end{tabular}

mean and variance values of $Z$ and $\Lambda$ provided in table 2 the mean value of the progress parameter is 0.22 for model A, whereas it is very close to zero for model $\mathrm{B}$. The values of the mixture fraction are very close to each other. Figure 15 shows the corresponding mixture-fraction distributions evaluated by the $\beta$-function and by the $P_{S M L, 2} \mathrm{PDF}$. The two distribution of $\Lambda$ (not shown) are both Dirac distributions since, due to the zero variance, the $\beta$ function of model B collapses on a $\delta$-function centred at $\widetilde{\Lambda}=0.008$.

At the second point on the axis of the burner $\left(x / d_{r e f}=27\right)$, the reaction is active for both models, as shown by the progress parameter values in table 2, which indicates that the combustion rate is higher for model A. Model B also provides lower values for the mean and variance of the mixture fraction. The PDF of $Z$ and $\Lambda$ are shown in figure 16. It appears that the $P_{S M L, 2}$ function is close to the $\beta$-distribution due to the low variance. The main differences between the two models are clearly observed in the right panel of figure 16 where the $P_{S M L, 2}(\Lambda)$ and the $\delta(\Lambda)$ are shown in order to put in evidence the dramatic simplification made when assuming the Dirac distribution for the progress parameter.

Finally, considering the third point close to the burner $\left(x / d_{r e f}=0.85, y / d_{r e f}=0.8\right)$, table 2 shows that a large difference in the progress-variable mean value exists between the two models. In fact, model $\mathrm{A}$ provides $\widetilde{\Lambda}=0.49$, indicating that combustion is active, whereas the mean value of $\Lambda$ for model $\mathrm{B}$ is close to zero. Figure 17 shows the corresponding PDF of $Z$ and $\Lambda$. Again, the $P_{S M L, 2}(Z)$ is very close to the $\beta$-distribution (left panel), whereas the $P_{S M L, 2}(\Lambda)$ maintains a smoother behaviour with respect to the $\delta$ function adopted for model A (right panel). The latter difference can be considered a reasonable motivation for the smoother behaviour of the temperature predicted close to the burner by model B (see figures 8 and 9.). 


\section{Conclusions}

This paper presents a statistical more likely distribution (SMLD) approach for the evaluation of the presumed probability density function (PDF) in flamelet progress variable (FPV) models for non-premixed combustion. The FPV model is employed in conjunction with the Reynolds averaged Navier-Stokes (RANS) equations. The proposed SMLD model is built evaluating the most probable joint distribution of the mixture fraction and of the progress variable and its adequateness and feasibility are discussed in comparison with the standard FPV model. The SMLD model relies on a more robust theoretical basis with a substantially unchanged computational cost. Although the classical formulation of the FPV approach is based on the lowMach-number assumption, we solve the total energy conservation equation to improve the computation of compressible reacting flows. The performance of the two FPV models are discussed by analysing the results of the simulation of a supersonic $H_{2}$-Air combustion studied at the NASA Langley Research Center. This analysis shows that the FPV-SMLD model provides an effective improvement over the standard approach and is able to properly describe the flame structure in good agreement with the results obtained by highly resolved LES with detailed chemistry. In fact, the numerical results correctly predict the presence of the three characteristic regions of supersonic flames: the autoignition, the stabilization and the combustion regions. Moreover, the FPV-SMLD model can correctly evaluate the lift-off of the flame whereas the standard model is not able to predict the combustion kinetic with sufficient accuracy, providing a sudden ignition of the mixture close to the burner inlet. A detailed analysis of the PDF distributions at several points of the computational domain is provided in order to quantify and explain the differences between the two models. This work has shown that indeed evaluating the PDF using the SMLD approach allows one to improve the accuracy of the simulation of a reacting supersonic flow in the framework of RANS equations. However, several aspects should be considered to enhance this analysis, such as: the effects of compressibility deserve to be analyzed in more details employing, for instance, a compressible FPV model; the effects of the hypothesis that the mixture fraction and the progress variable are not statistically independent have to be studied; the influence of the proposed FPV-SMLD model in the LES framework should be investigated. However, all this topics go beyond the aim of the present paper and will be the subject of our future work.

\section{Acknowledgements}

This research has been supported by grant n. PON03PE_00067_6 APULIA SPACE. The authors are very grateful to M. D. de Tullio for his support in the software development and to T.S. Cheng for providing the experimental data used as reference. Then, the authors wish to thanks P. Boivin for providing numerical LES results used as reference and for the interesting and useful discussion.

\section{References}

[1] D. Cecere, E. Giacomazzi, A. Ingenito, A review on hydrogen industrial aerospace applications, International Journal of Hydrogen Energy 39 (2014) 10731-10747.

[2] T. S. Cheng, J. A. Wehrmeyer, R. W. Pitz, Simultaneous temperature and multispecies measurement in a lifted hydrogen diffusion flame, Combust. Flame 91 (3) (1992) 323-345.

[3] M. Stöllinger, S. Heinz, Evaluation of scalar mixing and time scale models in PDF simulations of a turbulent premixed flame, Combustion and Flame 157 (9) (2010) 1671-1685.

[4] S. Lu, J. Fan, K. Luo, High-fidelity resolution of the characteristic structures of a supersonic hydrogen jet flame with heated co-flow air, International Journal of Hydrogen Energy 37 (2012) 3528-3539.

[5] T. Jin, K. Luo, S. Lu, J. Fan, DNS investigation on flame structure and scalar dissipation of a supersonic lifted hydrogen jet flame in heated coflow, International Journal of Hydrogen Energy 38 (23) (2013) 9886-9896.

[6] D. Cecere, A. Ingenito, E. Giacomazzi, L. Romagnosi, C. Bruno, Hydrogen/air supersonic combustion for future hypersonic vehicles, International Journal of Hydrogen Energy 36 (2011) 11969-11984.

[7] P. Boivin, A. Dauptain, C. Jimnez, B. Cuenot, Simulation of a supersonic hydrogen-air autoignition-stabilized flame using reduced chemistry, Combustion and Flame 159 (4) (2012) 1779-1790.

[8] F. Williams, Detailed and reduced chemistry for hydrogen autoignition, Journal of Loss Prevention in the Process Industries 21 (2) (2008) 131-135.

[9] L. V. Bezgin, V. I. Kopchenov, A. S. Sharipov, N. S. Titova, A. M. Starik, Evaluation of prediction ability of detailed reaction mechanisms in the combustion performance in hydrogen/air supersonic flow, Combust. Sci. Technol 185 (2013) $62-94$. 
[10] U. Maas, S. Pope, Simplifying chemical kinetics- intrinsic low-dimensional manifolds in composition space, Combust. Flame 88 (1992) 239-264.

[11] C. Pierce, Progress-variable approach for large-eddy simulation of turbulent combustion, PhD Thesis, Stanford University (2001).

[12] O. Gicquel, N. Darabiha, D. Thevenin, Laminar premixed hydrogen/air counterflow flame simulations using flame prolongation of ILDM with differential diffusion, Proc. Combust. Inst. 28 (2000) 1901-1908.

[13] J. V. Oijen, L. D. Goey, Modelling of premixed laminar flames using flamelet-generated manifolds, Combust. Sci. Technol. $161(2000)$ 113-137.

[14] H. Pitsch, N. Peters, A consistent flamelet formulation for non-premixed combustion considering differential diffusion effects, Combustion and Flame 114 (12) (1998) $26-40$.

[15] D. Carbonell, C. Perez-Segarra, P. Coelho, A. Oliva, Flamelet mathematical models for non-premixed laminar combustion, Combustion and Flame 156 (2) (2009) $334-347$.

[16] E. Knudsen, H. Pitsch, A general flamelet transformation useful for distinguishing between premixed and non-premixed modes of combustion, Combustion and Flame 156 (3) (2009) $678-696$.

[17] E. Knudsen, H. Pitsch, Capabilities and limitations of multi-regime flamelet combustion models, Combustion and Flame 159 (1) (2012) $242-264$.

[18] M. Oevermann, Numerical investigation of turbulent hydrogen supersonic combustion is a scramjet using flame let modeling, Aerosp. Sci. Technol. 4 (2000) 463-480.

[19] L. Hou, D. Niu, Z. Ren, Partially premixed flamelet modeling in a hydrogen-fueled supersonic combustor, International Journal of Hydrogen Energy 39 (17) (2014) 9497 - 9504.

[20] R. Pecnik, V. E. Terrapon, F. Ham, G. Iaccarino, H. Pitsch, Reynolds-Averaged NavierStokes Simulations of the HyShot II Scramjet, AIAA Journal 50 (8) (2012) 1717-1732.

[21] A. Saghafian, V. E. Terrapon, H. Pitsch, An efficient flamelet-based combustion model for compressible flows, Combustion and Flame 162 (3) (2015) $652-667$.

[22] N. Peters, Turbulent combustion, Cambridge University Press, 2000.

[23] C. D. Pierce, P. Moin, Progress-variable approach for large-eddy simulation of non-premixed turbulent combustion, J. Fluid Mech. 504 (2004) 73-97.

[24] S. Pope, PDF methods for turbulent reactive flows, Prog. Energy Combust. Sci. 11 (1985) 119-192.

[25] M. Ihme, H. Pitsch, Prediction of extinction and re-ignition in non-premixed turbulent flames using a flamelet progress variable model. 1 A priori study and presumed PDF, Combust. Flame 155 (2008) 70-89.

[26] M. Ihme, L. Shunn, J. Zhang, Regularization of reaction progress variable for application to flamelet-based combustion models, J. Comput. Phys. 231 (2012) 7715-7721.

[27] A. Najafi-Yazdi, B. Cuenot, L. Mongeau, Systematic definition of progress variables and intrinsically low-dimensional, flamelet generated manifolds for chemistry tabulation, Combust. Flame 159 (2012) 1197-1204.

[28] Y.-S. Niu, L. Vervisch, P. D. Tao, An optimization-based approach to detailed chemistry tabulation: Automated progress variable definition, Combust. Flame 160 (2013) 776785.

[29] H. Pitsch, M. Chen, N. Peters, Unsteady flamelet modelling of turbulent hydrogen/air diffusion flames, Proceeding of the Combustion Institute 27 (1998) 1057-1064.

[30] J. S. Kim, F. A. Williams, Structures of flow and mixture-fraction fields for counterflow diffusion flames with small stoichiometric mixture fractions, Journal of Applied Mathematics 53 (1993) 1551-1566.

[31] N. Peters, Laminar diffusion flamelet models in non-premixed turbulent combustion, Prog. Energy Combust. Sci. 10 (1984) 319-339.

[32] M. Ihme, C. M. Cha, H. Pitsch, Prediction of local extinction and re-ignition effects in non-premixed turbulent combustion using a flamelet/progress variable approach., Proc. Combust. Inst. 30 (2005) 793-800.

[33] M. Ihme, H. Pitsch, Prediction of extinction and re-ignition in non-premixed turbulent flames using a flamelet progress variable model. 2 Application in LES of Sandia Flames D and E, Combust. Flame 155 (2008) 90-107.

[34] R. De Meester, B. Naud, B. Merci, A priori investigation of pdf-modeling assumptions for a turbulent swirling bluff body flame ('sm1'), Combustion and Flame 159 (11) (2012) 3353-3357.

[35] S. Mukhopadhyay, J. Abraham, Evaluation of an unsteady flamelet progress variable model for autoignition and flame development in compositionally stratified mixtures, Phys. Fluids 24 (2012) 075115

[36] A. Coclite, G. Pascazio, P. De Palma, L. Cutrone, M. Ihme, An SMLD joint PDF model for turbulent non-premixed combustion using the flamelet progress-variable approach, Flow, Turbulence and Combustion 95 (1) (2014) 97-119.

[37] L. Cutrone, P. De Palma, G. Pascazio, M. Napolitano, A RANS flamelet-progress-variable method for computing reacting flows of real-gas mixtures, Comput. Fluids 39 (2010) 485-498.

[38] A. W. Cook, J. J. Riley, A subgrid model for equilibrium chemistry in turbulent flows, Phys. Fluids 6 (1994) $2868-2870$.

[39] J. Jimenez, A. Linan, M. M. Rogers, F. J. Higuera, A priori testing of subgrid models for chemically reacting non-premixed turbulent shear flows, J. Fluid Mech. 349 (1997) 149-171.

[40] C. Wall, B. J. Boersma, P. Moin, An evaluation of the assumed beta probability density function subgrid-scale model for large eddy simulation of non-premixed, turbulent combustion with heat release, Phys. Fluids 12 (2000) 2522-2529.

[41] D. A. Schwer, Numerical study of unsteadiness in non-reacting and reacting mixing layers, PhD Thesis, The Pennsylvania State University (1999).

[42] P. Saxena, F. Williams, Testing a small detailed chemical-kinetic mechanism for the combustion of hydrogen and carbon monoxide, Combustion and Flame 145 (1-2) (2006) 316-323.

[43] H. Pitsch, Flamemaster v3.3. A C++ computer program for 0d combustion and 1d laminar flame calculations.

[44] J. L. Steger, R. F. Warming, Flux vector splitting of the inviscid gas-dynamic equations with applications to finite difference 
methods, J. Comput. Phys. 40 (1981) 263-293.

[45] T. H. Pulliam, D. S. Chaussee, A diagonal form of an implicit factorization algorithm, J. Comput. Phys. 39 (1981) $347-363$.

[46] P. E. O. Buelow, D. A. Schwer, J.-Z. Feng, C. L. Merkle, D. Choi, A preconditioned dual time diagonalized ADI scheme for unsteady computations, in: AIAA Proceedings, 1997.

[47] F. Menter, C. Rumsey, Assessment of two-equation turbulence models for transonic flows, in: 25 ${ }^{\text {th }}$ AIAA Fluid Dynamics Conference, AIAA, Colorado Springs, CO, 1994.

[48] T. Cheng, J. Wehrmeyer, R. Pitz, O. Jarrett Jr., G. Northam, Raman measurement of mixing and finite-rate chemistry in a supersonic hydrogen-air diffusion flame, Combustion and Flame 99 (1) (1994) 157-173.

[49] T. Bridel-Bertomeu, P. Boivin, Explicit chemical timescale as a substitute for tabulated chemistry in a h2o2 turbulent flame simulation, Combustion Science and Technology (2014) null.

[50] Y. Moule, V. Sabelnikov, A. Mura, Highly resolved numerical simulation of combustion in supersonic hydrogenair coflowing jets, Combustion and Flame (0) (2014) -. 